\title{
Terminalidade humana na UTI: reflexões sobre a formação profissional e ética diante da finitude
}

\author{
Human terminality in ICU: \\ reflections on professional training and ethics in the face of finitude
}

Luana F. de Almeida*

\section{Resumo}

Profissionais de saúde convivem, no contexto da unidade de terapia intensiva (UTI), mais diretamente com os pacientes graves e seus familiares e lidam constantemente com a presença de suporte biotecnológico a órgãos vitais, isto é, entre possibilidades de manutenção da vida e enfretamento da morte. Diante dessa realidade, surgem profundas tensões emocionais relacionadas às expectativas e frustrações advindas da luta cotidiana contra a finitude humana. No entanto, observa-se, no processo de formação dos diferentes profissionais atuantes em terapia intensiva, a ausência de qualquer abordagem nas diferentes disciplinas que discutam aspectos cognitivos e afetivos relacionados ao processo da morte. Ainda que a reflexão sobre os aspectos envolvidos no melhor preparo para lidar com a morte seja estimulada por diversos autores, é inegável que as escolas de formação em saúde ainda enfrentam dificuldades para assumir o compromisso educacional com essa temática. Tal realidade demonstra a falta de repertórios adequados para lidar com decisões inerentes à atuação dos profissionais de saúde: a determinação de quem ocupa um leito - de quem vive e de quem morre - corresponde e resgata dilemas éticos a serem tratados. Isto implica uma discussão multidisciplinar no ambiente de formação e de atuação, o que explica a crescente presença de profissionais da psicologia e da saúde mental nas UTIs, abrindo espaço para a discussão da morte com os membros da equipe de saúde, que frequentemente lidam com sentimentos de perda, sofrimento, dor e fracasso, mas também com os pacientes e seus familiares. Nesse panorama, é possível perceber a importância, complexidade e especificidades das situações vividas por profissionais de saúde e pacientes no contexto de uma UTI. A sistematização de discussões relacionadas ao tema da morte pode constituir um meio eficaz de articulação entre a prática vivenciada e as perspectivas pessoal e profissional de médicos, enfermeiros, fisioterapeutas, entre outros, que testemunham ou testemunharão o complexo morrer humano.

Descritores: Morte; Educação; Unidades de terapia intensiva; Bioética. 


\section{Abstract}

In an intensive care unit environment (ICU), healthcare professionals live in straight contact with critically ill patients and their relatives, also continuously dealing with the presence of biotechnological support to vital organs, possibilities of life maintenance, and coping with death. Given this reality, deep emotional tensions arise from expectations and frustrations stemming from the daily fight against the supposed enemy - human finitude. However, throughout the process of qualification of the different intensive care therapy professionals, the absence of any approach from various disciplines to discuss cognitive and affective aspects related to the process of death is evidenced. Even if the reflection about the factors involved in the best preparation to cope with death is encouraged by many authors, it is undeniable that health training institutions still struggle to take over an educational commitment with this subject. Such reality evidences the lack of appropriate repertoire to deal with decisions inherent to the performance of healthcare professionals: the determination of who lies on a bed, of those who live or die corresponds to ethical dilemmas to be addressed. That entails a multidisciplinary discussion, both in health training institutions and in healthcare work environments - which explains the growing presence of psychology and mental healthcare professionals in ICUs - making room for talking about death with healthcare team members who often deal with feelings of loss, pain, and failure, but also with patients and their relatives. In this scenario, it is possible to realize the importance, complexity and specificity of situations experienced by healthcare professionals and patients within an ICU context. The systematization of discussions related to death can be an effective alternative to articulate hands-on experiences with personal and professional perspectives from physicians, nurses, physiotherapists, among others, who witness or will witness the complex death of human beings.

Keywords: Death; Education; Intensive care units; Bioethics.

\section{Introdução}

No desempenhar do seu papel de salvar vidas, os profissionais de saúde vivenciam, no contexto específico da UTI, possibilidades de sucesso ou fracasso, pautando suas ações em complexas decisões potencializadas pelas características desse ambiente. Além da presença de suporte a órgãos vitais e possibilidades de manutenção da vida, esses profissionais convivem mais diretamente com os pacientes graves e seus familiares, sendo, também, frequentemente questionados por esses sobre a evolução da doença, perspectivas de tratamento e expectativas de vida dos pacientes. Essas situações podem criar circunstâncias angustiantes relacionadas com a proximidade da finitude humana e a impossibilidade de dominá-la. Assim, as manifestações presentes nos profissionais se relacionam às exigências ligadas à sua função e às maneiras pelas quais essas mesmas exigências são sentidas por cada um deles. São comuns os sentimentos de culpa relacionados à incapacidade de evitar a morte e ao alto grau de exigência de suas próprias responsabilidades. ${ }^{1}$

Observa-se que as UTIs, ao mesmo tempo em que correspondem a setores nos quais a realização de procedimentos e cuidados é intensa, também correspondem a espaços naturalmente mobilizadores de emoções e sentimentos que, frequentemente, se manifestam de forma muito expressiva. O estresse atinge a todos, ou seja, pacientes, família, médicos, enfermeiros, fisioterapeutas e outros profissionais. Esses elementos vivem um clima de constante tensão, resultante do contato com a iminência de algum risco e/ ou desequilíbrio no estado dos pacientes, das demandas e solicitações que devem ser respondidas com presteza, da realização de grande número de procedimentos complexos e do ritmo intenso de trabalho. Trata-se de uma vivência simultânea de onipotência e impotência que pode gerar nos intensivistas sentimentos complexos e ambíguos de segurança e insegurança, certeza e incerteza, frente à tarefa de salvar vidas.

Desta maneira, percebe-se que o dia a dia na UTI se mostra polarizado por situações an- 
tagônicas na qual, às vezes, é possível salvar ou curar, e em outras, prorroga-se o sofrimento e confronta-se a presença da morte. Os profissionais, muitas vezes, são submetidos às pressões e dilemas éticos quanto à tomada de decisões em momentos críticos, como a questão sobre o prolongamento ou não da vida em casos sem prognóstico. Toda essa demanda gera a necessidade de uma formação específica para lidar com situações nas quais, rotineiramente, os profissionais de terapia intensiva estão envolvidos.

\section{O trabalho na UTI}

O que representa ou assinala uma ameaça à vida exige uma intervenção imediata dos profissionais intensivistas, como é o caso de uma diminuição nos batimentos cardíacos e outros sinais clínicos (um sangramento evidente, por exemplo). A noção de prioridade é estabelecida por esses profissionais e costuma ser formada a partir de uma apreciação baseada em protocolos ensinados nos manuais de rotina, que descrevem as etapas dos procedimentos terapêuticos a serem seguidos para os problemas de saúde mais comuns no atendimento ao paciente com risco de vida e/ou iminência de morte.

Pitta $^{2}$ assinala a variedade de situações extremas às quais os intensivistas estão intermitentemente submetidos: “(...) a solicitação constante de decisões rápidas e precisas; a necessidade de um grande número de informações serem processadas num curto espaço de tempo; a imensa responsabilidade em ter "uma vida nas mãos"; a tarefa desumana de "selecionar" quem usa este ou aquele equipamento; as situações de intercorrências inesperadas no quadro clínico dos pacientes, proporcionando um estado de alerta permanente; e o contato com a morte em tarefa diária”.2

No meio de um complexo conjunto de ações, na UTI, rotina e urgência se alternam. Notam-se momentos nos quais a tensão cresce ou o grupo profissional que ali atua entra em dinâmicas muito especiais e características profissionais e pessoais se manifestam. Como, por exemplo: na chegada de um paciente em estado crucial que exige decisões e ações de alta importância; no conflito de interpretação de uma situação: "deve ser feito isto ou aquilo?"; na interferência da família que quer ver o paciente e sofre impactos pelo seu estado de saúde; na não aceitação por parte do paciente de grave situação; nos agravamentos do quadro clínico e/ou na percepção de que algo vai muito mal a despeito de todos os cuidados; e na iminência da morte ou morte efetiva.

Na UTI, os profissionais de saúde, ocupando lugar e papéis distintos, participam do atendimento ao paciente com risco de vida e/ ou iminência de morte. A interdependência e a interligação de suas apreciações e avaliações desenham uma corrente dinâmica de decisões e ações que, afinal, determinam a qualidade dos cuidados prestados em termos de rapidez e eficácia. Isto, de fato, caracteriza o trabalho na UTI como produto da equipe multiprofissional intensivista, mas não retira o sentimento de responsabilidade profissional individual e a eventual culpa oriunda de alguma situação que possa ter saído do seu controle.

De fato, no processo de formação de profissionais de saúde, a terminalidade humana não é tema de ação pedagógica para que se desenvolvam comportamentos adequados em relação à situação da morte de um paciente ou de um paciente com diagnóstico grave. Observa-se, no processo de formação nos diferentes profissionais atuantes em terapia intensiva, a ausência de qualquer abordagem nas diferentes disciplinas que discutam aspectos cognitivos e afetivos relacionados ao processo da morte e do morrer. Ainda que a reflexão sobre os distintos aspectos envolvidos no ensino da morte seja estimulada por diversos autores, é inegável que as escolas de formação em saúde ainda enfrentam dificuldades para assumir o compromisso educacional com essa temática. Poucas oferecem disciplinas que tratem do tema da morte e, quando o fazem, geralmente são disciplinas eletivas ou de carga horária restrita. ${ }^{3,4,5,6,7}$ 


\section{Aspectos da finitude humana na UTI}

O processo de tomada de decisões médicas relativas à vida e morte dos pacientes de UTI se revela de extrema complexidade na avaliação prática do intensivista sobre o paciente. Através da tecnologia, consegue-se hoje postergar, prorrogar a vida. A ciência médica atingiu um patamar de desenvolvimento tecnológico que permite certo domínio e regulação da morte, em outras palavras, uma "domesticação" da morte. ${ }^{8}$

O desenvolvimento técnico das últimas décadas possibilitou suporte a órgãos humanos e funções vitais. Cada vez mais, realizam-se pesquisas voltadas para a obtenção de aparelhos que possibilitem manter vivos os homens. Na UTI, a morte, processo natural da vida, pode ser levada a ocorrer fora do tempo real, projetada para o futuro através de aparelhos que conseguem, contemporaneamente, prolongar a vida com a ilusão de dar crédito à imortalidade.

O paciente, nesse contexto, é constantemente monitorizado. Não é mais um ser humano autônomo, as máquinas fazem parte dele. Sem elas, em alguns momentos, ele não seria capaz de viver. O objetivo é colocar o paciente com alto risco de vida e/ou iminência de morte em um ambiente em que suas funções vitais são continuamente monitoradas, e onde a intervenção salvadora possa ser feita rapidamente, de forma concentrada e intensa, em um único lugar. Esse progresso da medicina intensiva se deve ao avanço da tecnologia biomédica.

Assim, nas UTIs, bem como em outros setores do hospital, o objetivo é suplantar a morte. Há regras e funções estipuladas, predeterminadas para o alcance da vitória sobre o pretenso inimigo. Os atarefados profissionais envolvidos na luta contra a morte, muitas vezes, assustam -se com seus limites e impotência. Nesse contexto, a eficiência técnica da máquina é colocada à disposição do paciente, significando a decisão de que esta acabou de penetrar nos limites biológicos da morte. Desta forma, a morte deixa de ser pontual, definitiva e privada, passando a se integrar nas políticas de intervenção sobre a vida.

Nesse espaço, a partir dos avanços da biomedicina, a morte passa a ser encefálica, biológica e celular. A finitude humana é dividida, parcelada numa série de pequenas etapas, como afirma Airès, ${ }^{8}$ entre as quais, definitivamente, não se sabe qual é verdadeiramente a morte, aquela em que se perde a consciência ou aquela em que se perde a respiração. Essa subdivisão, como refere Kovács, ${ }^{9}$ torna-se relevante quando envolve a polêmica dos transplantes, em que pacientes com morte cerebral são mantidos vivos para que os órgãos possam ser retirados e utilizados em uma nova vida. O momento da morte ou a interrupção da vida, nesses casos, passa a ser acordado entre os médicos, familiares e outros profissionais.

Compreende-se, desse modo, que através do emprego da tecnologia médica para a sustentação do viver, com a criação e utilização do respirador artificial, ocorreram profundas alterações, tanto no processo de morrer, quanto no próprio conceito de morte. O conceito de morte cerebral, como descrito anteriormente, é articulado ao transplante de órgãos. ${ }^{10}$ A morte de distintas partes do corpo de um indivíduo é possível através da tecnologia médica. Assim, a imagem da morte com o tradicional esqueleto com foice foi substituída, no século XX, pela imagem de um paciente internado na UTI, conectado a tubos e cercado de aparelhos que mantêm a vida. Este modelo de morte é denominado "morte moderna", medicalizada. ${ }^{8}$

Com tantos desequilíbrios, incertezas e paradoxos, na UTI facilmente se passa da luta pela vida à morte resignada; do desafio que se impõe em manter o paciente vivo ao medo da sua própria morte. Nada é permanente nesse contexto. Trata-se de um espaço ambíguo, no qual se procura controlar, através mesmo da negação e isolamento, o imprevisível.

\section{Bioética na UTI}

Observa-se que a morte na UTI se encaixa no que refere Airès, ${ }^{8}$ ou seja, a morte, no con- 
texto hospitalar, consiste em fenômeno técnico, causado pela parada dos cuidados, mais ou menos declarada, por decisão do médico e da equipe de saúde. São eles os donos da morte, do seu momento e de suas circunstâncias. A variabilidade da duração da morte é estabelecida pelos progressos da biomedicina. Dentro de certos limites, pode-se abreviá-la ou estendê-la, dependendo da decisão do médico e dos equipamentos disponíveis. Conjugadas à manutenção da vida surgem novas questões: quando podem ou devem ser desligados os aparelhos de manutenção da vida? Quais os critérios na tomada de decisões? Quais os atores envolvidos e os papéis desempenhados no processo decisório?

Os problemas éticos começaram a existir com os pacientes que não têm chance de se recuperar facilmente e ficam muito tempo na UTI, com suas funções vitais mantidas artificialmente. Esse fato não havia sido previsto na ideia fundamental da UTI, cuja filosofia é cuidar muito intensamente, por pouco tempo. Para esses pacientes, imersos em contradições e dilemas da tecnologia e da ética, a vida é discutível. Os custos explodem. Uma estadia em uma UTI apresenta um alto ônus financeiro e não resulta necessariamente em cura ou alta.

A bioética, então, surge na UTI como forma de reflexão para os profissionais intensivistas, associada à ação integrada da tecnologia do saber. A decisão de quem ocupa um leito, de quem vive e quem morre corresponde, certamente, a uma realidade a ser tratada. A morte passa a ser discutida. Surgem debates acerca dos termos eutanásia, ortotanásia e distanásia. Como indica Torres, ${ }^{11}$ a morte correta (ortotanásia), em seu tempo certo, nem abreviada (eutanásia), nem prolongada no tempo (distanásia), confronta médicos e enfermeiros num imperativo ético de reflexão sistemática sobre as posições assumidas por estes quanto aos tempos de morte dos pacientes na UTI.

Kovács, ${ }^{4}$ psicóloga, estudiosa dos assuntos da morte e do morrer, menciona que ao se priorizar no hospital, em especial na UTI, o salvar o paciente a qualquer custo, a ocorrência de morte pode fazer com que o trabalho de médicos e enfermeiros, nesse contexto, seja percebido como frustrante, desmotivador e sem significado. A psicóloga acrescenta que esta percepção pode ser agravada quando os procedimentos médicos a serem realizados, fora de possibilidade de cura, não são compartilhados com toda a equipe. Por outro lado, não conseguir evitar ou adiar a morte pode trazer ao profissional a vivência de seus limites, $o$ que, em algumas vezes, pode ser extremamente doloroso.

Situações geradoras de grandes dilemas éticos podem levar pacientes (se conscientes), familiares e profissionais a se depararem com a necessidade de tomada de decisão, no que concerne ao prolongamento ou interrupção das medidas terapêuticas. Delimitar o tratamento e definir ações correspondem a difíceis decisões, nem sempre concordantes e uniformes.

\section{A morte como um assunto a ser discutido}

Estudiosos do assunto, tanto na literatura nacional como internacional, concordam sobre a necessidade de se discutir amplamente todas as alternativas terapêuticas possíveis para a solução dos dilemas éticos inerentes ao tratamento intensivo e a terminalidade humana. Recomenda-se que a discussão seja interdisciplinar, agregando tantos profissionais quanto possível. $^{12,13}$

$\mathrm{Na}$ prática, no entanto, tal procedimento ainda é pouco observado e, na maioria das vezes, o médico é levado a decidir isolada e unilateralmente, não compartilhando opiniões devido à falta de comunicação entre os profissionais. Percebe-se, também, que a decisão de se interromper ou prolongar determinado tratamento nem sempre é consensual e sustentada pelos diferentes intensivistas da mesma instituição. Dessa forma, não é incomum que em plantões diferentes, um outro médico retome o tratamento anterior, motivado por suas convicções pessoais, criando um círculo vicioso de difícil solução e que reflete a falta de diálogo entre a 
própria equipe médica. Tais condutas, indesejáveis e contraditórias, não só confundem profissionais e familiares, como podem aumentar ou trazer falsas expectativas em relação à evolução clínica do paciente.

Diante disso, surgem algumas questões: Como comunicar aos pacientes e aos seus familiares o agravamento da doença e a proximidade da morte? Como lidar com pacientes que estejam apresentando intensa expressão de sentimentos (medo, raiva e tristeza)? Como desenvolver o tratamento de pacientes sem possibilidade de cura (aprofundando a diferença entre curar e cuidar)? Como abordar a família quando há aproximação da morte e como acolher os sentimentos presentes nesta situação? Como lidar com a expressão do desejo de morrer por parte do paciente ou da família, que não suporta ver tanto sofrimento? Como explicar para os familiares uma mudança de conduta radicalmente oposta? O que o intensivista pode falar ou não a respeito de uma decisão da qual ele não participou? O que seria eticamente correto?

\section{Conclusão}

Percebe-se que a discussão dos temas relacionados à vida, à morte e ao processo de morrer dentro das UTIs é de fundamental importância. Assim, pode-se explicar como profissionais da psicologia e da saúde mental começam a fazer parte das equipes nas UTIs, não só abrindo espaço para a discussão da morte e do processo de morrer com os membros da equipe de saúde, que frequentemente lidam com sentimentos de perda, sofrimento, dor e fracasso, mas também com os pacientes e seus familiares.

Nesse panorama, é possível perceber a importância das situações vividas por profissionais de saúde e pacientes no contexto de uma UTI.E não é difícil argumentar sobre a necessidade de mais investigação em torno das nuances, questões e desafios à capacidade humana de vivenciar complexos momentos de envolvimento com a situação da proximidade do morrer humano. É indispensável a busca da ampliação da compreensão dos processos pessoais, emocionais, existenciais, profissionais e institucionais aí envolvidos.

Não obstante, esse processo de maior compreensão e de reconhecimento da necessidade de lidar com todas as questões que surgem e que envolvem o processo de morrer deve ser trazido à tona nos espaços de formação dos profissionais de saúde. A sistematização de discussões relacionadas ao tema aqui exposto, em diferentes cenários, pode constituir um meio eficaz de articulação entre a prática vivenciada e as expectativas pessoais e profissionais de médicos, enfermeiros, fisioterapeutas, entre outros, que testemunham ou que testemunharão o complexo morrer humano.

\section{Referências}

1. Bretãs JRS, Oliveira JR, Yamaguti L. Reflexões de estudantes de enfermagem sobre morte e o morrer. Rev Esc Enferm USP. 2006;40(4):477-83.

2. Pitta A. Hospital: dor e morte como ofício. São Paulo: Hucitec; 1994. p.4.

3. Viana A, Picelli H. O estudante, o médico e o professor de medicina perante à morte e o paciente terminal. Rev Bras Educ Med. 1998;44(1):21-7.

4. Kovács MJ. Educação à morte: desafio na formação de profissionais de saúde e educação. São Paulo: Casa do Psicólogo; 2003. p.117.

5. Sousa DM, Soares EO, Costa KMS, Pacífico ALC, Parente ACM. Vivência da enfermeira no processo de morte e morrer dos pacientes oncológicos. Texto \& Contexto Enfermagem. 2009 Jan/Mar;18(1):41-7.

6. Silva GSN, Ayres JRCM. O encontro com a morte: à procura do mestre Quíron na formação médica. Rev Bras Educ Med. 2010 Oct/Dec;34(4):487-96.

7. Azeredo NSG, Rocha CF, Carvalho PRA. O enfrentamento da morte e do morrer na formação de acadêmicos de Medicina. Rev Bras Educ Med. 2011 Jan/Mar;35(1):37-43.

8. Ariés P. O homem diante da morte. Rio de Janeiro: Ediouro; 2003. p.639.

9. Kovács MJ. Educação à morte: temas e reflexões. São Paulo: Casa do Psicólogo; 2003. p.71.

10. Lock M. Twice dead: organs transplants and the reinvention of death. Berkeley: University of California Press; 2001. p.233. 
11. Torres WC. A Bioética e a Psicologia da Saúde: Reflexão sobre questões de vida e morte. Psicologia, Reflexao E Critica 2003;16(3):475-82.

12. Orlando JMC. Aspectos relacionados ao paciente: direitos do cidadão em estado crítico. In: _. UTI muito além da técnica...a humanização e a arte do intensivismo. São Paulo: Atheneu; 2001. p.43-4.

13. Baggs JG. Two instruments to measure interdisciplinary bioethical decision making. Heart Lung 1993;22(6):542-7 